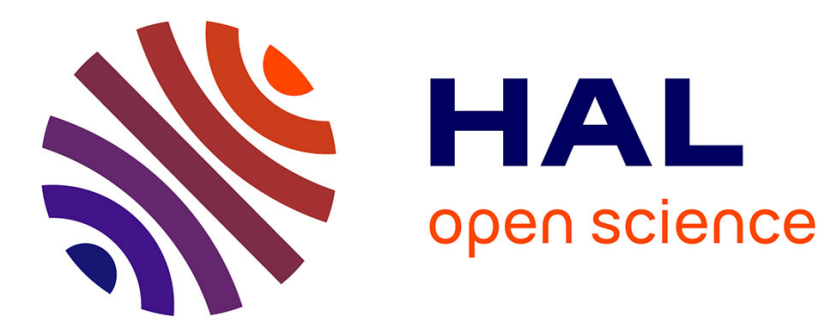

\title{
Rumeurs en scène et autres figures
}

Geneviève Mathon

\section{- To cite this version:}

Geneviève Mathon. Rumeurs en scène et autres figures: L'exemple de "Deux sur la balançoire". Visconti, 1958, Théâtre des Ambassadeurs. Ligeia, dossiers sur l'art, 2015, 141-144. hal-01365385

\section{HAL Id: hal-01365385 \\ https://hal.science/hal-01365385}

Submitted on 13 Sep 2016

HAL is a multi-disciplinary open access archive for the deposit and dissemination of scientific research documents, whether they are published or not. The documents may come from teaching and research institutions in France or abroad, or from public or private research centers.
L'archive ouverte pluridisciplinaire HAL, est destinée au dépôt et à la diffusion de documents scientifiques de niveau recherche, publiés ou non, émanant des établissements d'enseignement et de recherche français ou étrangers, des laboratoires publics ou privés. 


\section{RUMEURS EN SCENE ET AUTRES FIGURES \\ L'EXEMPLE DE DEUX SUR LA BALANÇOIRE \\ VISCONTI, 1958, THEATRE DES AMBASSADEURS}

\section{Geneviève Mathon}

\section{En guise de préambule ${ }^{1}$}

Le bruit ressortit à une mesure esthétique, à une appréciation que l'on pose selon l'angle de vue adopté ou encore subi. Au plan musical, le bruit a été, dans notre tradition occidentale, proscrit. «Les murs épais de l'architecture européenne ont structuré la musique, et ce, de la période du plain-chant à celle du sérialisme. En réalité, il serait possible d'écrire toute l'histoire de la musique européenne en se référant à la nature des murs qui l'ont vu évoluer $»^{2}$. De l'église à la salle de concert, en passant par les Cours et les salons, espaces clos et hautement civilisés, l'écoute s'est spécialisée, affinée et a défini des lieux de pouvoir. A la faveur des mutations du début du $\mathrm{XX}^{\mathrm{e}}$ siècle, le bruit a fait l'objet d'une conquête progressive et d'une intégration à l'intérieur même du langage musical qui en a admis l'existence, la puissance et enfin la valeur. La création musicale s'en est trouvée bouleversée, enrichie. Luigi Russolo en 1913, dans son «Manifeste futuriste» (Milan, 11 mars 1913) ${ }^{3}$, appréhende l'histoire de la musique selon une évolution progressive. L'intégration du bruit dans le champ musical est, selon lui, un progrès nécessaire, inévitable, plus encore il constitue un saut qualitatif (l'électricité a permis l'invention de machines productrices de bruits). Edgard Varèse reprend, rectifie les propos de Russolo, et musicalise le bruit : celui-ci ne sera «plus à part» (Russolo organisait des concerts bruitistes avec piano jouant une musique très conventionnelle assortie de bruits produits par les crépiteurs, les strideurs, les bourdonneurs et autres instruments-bruiteurs inventés); bruits et musiques se croisent, s'échangent, s'hybrident et inventent des organismes sonores hauts en timbres, en couleurs et en intensités.

Plusieurs facteurs, que nous décrirons brièvement, ont permis cette mutation à l'œuvre des le début du $\mathrm{XX}^{\mathrm{e}}$ siècle : d'une part, l'évolution du langage musical à proprement parler ; d'autre part, l'intrusion d' «ailleurs » sonores et musicaux qui vont élargir nos capacités

\footnotetext{
1. Danielle Mathieu-Bouillon et Olivier Morel m'ont permis l'accès à la bande-son réalisée par Fred Kiriloff. Ce travail qui mérite approfondissement n'aurait pu se faire sans leur aide. Je les remercie vivement et chaleureusement.

${ }^{2}$. R. Murray Schafer, «Musique / non-musique : intersections », in Jean-Jacques Nattiez (sous la direction de), Musiques, une encyclopédie pour le XXI siècle, vol. 1, Musiques du XX siècle, Actes Sud / Cité de la musique, 2003 pour la traduction française, p. 1190.

${ }^{3}$. Luigi Russolo, L'Art des bruits, Lausanne, L'Âge d'Homme, 1975, p. 35-43.
} 
d'écoute. L'environnement sonore, alors en pleine mutation, modifiait nos perceptions (Russolo décrit très bien ce mécanisme à l'échelle de la ville), et Debussy, Ravel et Varèse écoutaient les musiques des Expositions universelles. La découverte de la relativité des cultures autorisait la relativité des esthétiques. Ce processus n'aura de cesse de s'intensifier tout au long du $\mathrm{XX}^{\mathrm{e}}$ siècle via entre autres les moyens offerts par l'enregistrement. Enfin le langage musical sera profondément affecté par le mouvement expressionniste viennois des années 1900. L'attention portée à l' «expression brute et pulsionnelle » ${ }^{4}$ (selon les termes d'Adorno) libère le cri, introduit le désordre et l'irrationnel, et déstabilise l'édifice musical dans sa conception et son expression : «les premières œuvres atonales sont des 'procèsverbaux', au sens où en psychanalyse on parlerait de procès-verbaux de rêves $»^{5}$. Les travaux de Freud sont partie prenante de ces changements de paradigmes qui touchent la création littéraire et artistique. Le phénomène de l'inconscient est entré dans la création dont elle ne pourra désormais plus se départir.

Autrement dit, et dans un même mouvement, l'oreille accueille, ouvre et intègre d'autres modèles sonores. L'écoute devient lâcher-prise, comme si le compositeur admettait ou prenait la mesure de ce qu'il ne peut maîtriser. Ou plus simplement, comme si le compositeur, en s'ouvrant au monde sonore, lâchait prise également dans son rôle ou sa fonction de compositeur, devenant dès lors parfois un simple médiateur, un auditeur privilégié. C'est ce que Barthes définit comme la troisième écoute ${ }^{6}$.

Cette intégration musicale du bruit, cette conception d'une écoute qui s'ouvre à une polysémie de sensations est dans le domaine théâtral très sensible et pertinente : il n’y a pas que la musique à écouter, il y a aussi les paroles, les voix. Autrement dit un continuum s'organise depuis les voix qui sont dépositaires d'une histoire, d'un sens à véhiculer jusqu'aux bruit(age)s, en passant par les musiques d' «accompagnement ». Chacun des trois niveaux est agi, à son tour, selon un continuum. De l'assujettissement à un sens ou à un langage jusqu'à une relative autonomie, chaque niveau déploie une palette sonore riche et diversifiée.

En 1958, Luchino Visconti met en scène à Paris, aux Théâtre des Ambassadeurs, la pièce de William Gibson, Two for the Seesaw. La dimension sonore semble avoir gagné tous les niveaux de l'édifice ; depuis les voix des comédiens jusqu'aux musiques transmises par la radio en passant par la bande magnétique qui diffuse en continu. Conçue par Fred Kiriloff,

\footnotetext{
4. Theodor W. Adorno, Philosophie de la nouvelle musique [1949], Paris, Gallimard, 1962, p. 50.

5 . Ibid.

${ }^{6}$. Roland Barthes, «Ecoute », L'Obvie et l'obtus, Paris, Seuil, 1982, pp. 217-230. Paru pour la première fois dans l'Encyclopédie Einaudi, en 1976.
} 
elle est un des premiers exemples de l'emploi d'une bande-son au théâtre. En regard, la construction de la pièce répondra à des normes structurelles musicales.

La pièce produite à Broadway la même année a connu une série de métamorphoses par des changements de format et les différents protagonistes, auteurs, adaptateurs, traducteurs, metteurs en scène et réalisateurs qui s'en sont emparés. En décrire quelques étapes et ramifications est l'objet de la première partie. La seconde est consacrée à cette conjonction fabuleuse entre bruits et sons, musique et voix, que va inaugurer Luchino Visconti, aidé en cela par Fred Kiriloff. Au-delà de ce (triple) continuum qui ponctue et hiérarchise les éléments acoustiques en présence, une étape remarquable est franchie dans l'histoire du son au théâtre ; une nouvelle écoute est à l'œuvre.

\section{De Broadway à Paris...}

La pièce de William Gibson Two for the Seesaw est représentée au Booth Theatre entre le 16 janvier 1958 et le 31 octobre 1959. Drame en trois actes et neuf scènes, elle est la première incursion de Gibson sur Broadway. La production connaît 750 représentations. La mise en scène est signée Arthur Penn (1922-2007). Les rôles principaux sont tenus par Henry Fonda et Anne Bancroft qui fait ses débuts à Broadway. William Gibson a publié une chronique sur les vicissitudes de la réécriture imposée par Arthur Penn: The Seesaw Log $(1959)^{7}$. La structure de la pièce et son minimalisme ainsi que les choix de mise en scène induisent un drame très moderne sur l'impossibilité de communication au sein d'une grande métropole, où des gens de tous univers, se croisent, se rencontrent, se quittent. L'histoire en est cependant banale.

Jerry Ryan, avocat vivant dans le Nebraska, décide de quitter sa femme Tess avec laquelle il vit depuis 12 ans. Au début de leur mariage, le père de Tess l'avait introduit dans son cabinet d'avocats, gérant ainsi sa carrière et sa vie. La pièce débute lorsque Jerry arrivé depuis un mois à New-York, après avoir fui ce milieu qu'il juge bourgeois et étouffant, y rencontre Gittel Mosca (contraction de Moscavicz), jeune danseuse bohème, d'origine polonaise, souffrant d'un ulcère. Il veut prendre soin d'elle, commencer avec elle une nouvelle vie et oublier Tess. Les neuf scènes racontent l'histoire de cette rencontre entre deux êtres que tout sépare : milieu, profession, choix de vie. Un huis clos s'organise entre eux, chez l'un, chez l'autre ou les deux, avec New-York en toile de fond.

\footnotetext{
${ }^{7}$. Alfred A. Knopf, $1^{\text {ère }}$ édition (1959).
} 
La dramaturgie proposée par Arthur Penn défie la mise en scène de l'époque. La scène, coupée en deux, représente deux appartements distincts ; le téléphone les reliant devient un troisième personnage. Afin d'insuffler du mouvement à la mise en scène, Arthur Penn et George Jenkins, le décorateur et éclairagiste, ont élaboré deux plateaux tournants jumeaux. Ainsi, chaque scène est vue sous un angle de vue différent, et l'on se rapproche d'une vision cinématographique sans que jamais le rideau ne descende.

Trois ans plus tard (1962), Robert Wise, le réalisateur de West side story reprend la pièce, avec Robert Mitchum et Shirley MacLaine. Le découpage de la pièce de Gibson est repris dans le film de Wise. On retrouve les neuf scènes qui alternent entre les deux appartements. Le téléphone permet, comme dans la pièce de théâtre, de relier visuellement les deux lieux. Des scènes en extérieur sont ajoutées : on narre, on déplie ce qui avait été seulement suggéré dans la pièce. Le film rompt avec le minimalisme souhaité par Gibson. Les personnages qui y étaient seulement évoqués prennent chair, et les dialogues et situations se multiplient : il en résulte une comédie romantique à l'instar de Breakfast at Tiffany's (1961). La musique de André Prévin (1929), compositeur américain d'origine allemande, est très présente tout au long du film. Elle accompagne les errances de Robert Mitchum dans les rues de Manhattan. La chanson qui en sera extraite «Second chance » deviendra un standard de la musique jazz, enregistrée, entre autres, par Ella Fitzgerald. En 1973, la pièce est devenue la base pour une comédie musicale : Seesaw.

En France, c'est Louise de Vilmorin qui l'a traduite et adaptée à la scène ${ }^{8}$. Luchino Visconti en conçoit la mise en scène - sa première mise en scène parisienne - et les décors pour le Théâtre des Ambassadeurs ${ }^{9}$. Jean Marais lui avait proposé de la monter et lui avait

\footnotetext{
${ }^{8}$. Louise de Vilmorin (1902-1969) ${ }^{8}$ avait publié en 1951 Julietta et Madame de. Probablement a-t-elle été séduite par le personnage de Gittel, jeune femme elle aussi fantasque, légère et libérée. Louise de Vilmorin suit de près le texte de Gibson, elle fait quelques digressions avec notamment le jeu de lettres parfumées, bleues ou roses, que Tess envoie à Jerry.

${ }^{9}$. Le relevé de mise en scène du fonds de l'ART (Association de la Régie Théâtrale), conservé à la BHVP, consigne les dossiers suivants :
}

1 dossier $27 \times 21 \mathrm{~cm}$. Notes manuscrites de mise en scène sur copie dactylographiée. Coupures et variantes de texte. Description du décor. Cote : 4-TMS-03383 (RES)

Dossier technique ; 1958. 27 feuillets dactylographiés $27 \times 21 \mathrm{~cm}$. Indications de lumière. Indications de mise en état. Liste et minutage des musiques. Liste des meubles et accessoires. Contient 1 accessoire de la pièce : une notification fictive de divorce, en 2 feuillets dactylographiés, en anglais. Cote : 4TMS-03384 (RES)

Schéma d'implantation des éclairages. Croquis d'implantation de décor ; 1958. 1 feuillet manuscrit, 1 calque $63 \times 49 \mathrm{~cm}$. Cote : 1 -TMS-00008 (RES)

Indications de son, liste des accessoires ; 1959. 1 volume, 1 brochure, 1 feuillet $27 \times 21 \mathrm{~cm}$; croquis de placement et de mouvement des acteurs. Programme (Galas Karsenty). Cote : 4-TMS-03385 (RES) 
présenté Annie Girardot, sortie fraîchement de la Comédie-Française ${ }^{10}$. Le Tout-Paris est présent à la Première ; la pièce connaît un franc succès.

Luchino Visconti (1906-1976) avait monté, en janvier 1945, la première traduction italienne des Parents terribles de Cocteau au Teatro Eliseo à Rome. S'en étaient suivis La Machine à écrire du même Cocteau, Antigone d'Anouilh, Huis clos de Sartre. A partir de 1950, il fait connaître les auteurs américains, comme Arthur Miller (la Ménagerie de verre, Mort d'un commis voyageur) et Tennessee Williams (Un tramway nommé désir). Il aborde, dès 1954, la mise en scène d'opéras et dirige Maria Callas dans différents ouvrages : la Vestale de Spontini, la Traviata de Verdi, Anna Bolena de Donizetti et Iphigénie en Tauride de Gluck ces deux réalisations de 1957 marquent la fin de sa collaboration avec la Callas. Sa mise en scène de Deux sur la balançoire/Two for Seesaw se nourrit de toutes ces expériences d'adaptations, de mises en scène et d'un rapport intime à la musique. Il reprend de la pièce de Gibson le décor et le montage technique dans son ingéniosité et sa nouveauté.

Description du décor: "Le décor représente deux pièces apparemment contiguës, mais qui sont, en réalité, situés dans deux immeubles qu'une distance de plusieurs kilomètres sépare l'un de l'autre, à New-York. Le living-room de Jerry Ryan est placé sur le côté droit de la scène. C'est le très petit living-rom d'un morne appartement de deux pièces, au sixième étage d'un immeuble dans le bas quartier d'East-Side. Un divan, très étroit, et une chaise de cuisine, servant de table de chevet, en composent tout l'ameublement, et ce living-room, quand le rideau se lève, a l'aspect déprimant d'une pièce meublée de façon minable par un nouveau locataire qui vient tout juste d'y entrer. Le téléphone est posé sur le divan. Par la fenêtre, on voit les toits des maisons d'en face. Le mur de gauche, qui coupe la scène en deux, n'existe que partiellement de manière à ne pas nous masquer la vue sur l'autre pièce. Le living-room de gauche est celui de Gittel Mosca. C'est le living-room d'un appartement situé au dernier étage d'une vieille maison délabrée en plein centre de la ville. Le living-room, qui est placé en contre-bas de celui de Jerry, est plus grand et plus clair que le sien. Il y règne un agréable désordre et l'on sent que quelqu'un habite là depuis un certain temps. L'ameublement de cette pièce est d'un goût très ordinaire, mais il s'en dégage un sentiment humain réconfortant et chaud. Au-delà de ces deux intérieurs est figurée la ville de NewYork. »

La réalisation de Visconti : «Deux sur la balançoire présente de New-York une vision très séduisante - panorama d'immeubles illuminés dans la nuit, large vue de la ville au loin,

\footnotetext{
${ }^{10}$. Annie Girardot connaîtra dans la foulée une brillante carrière italienne avec, entre autres, Rocco et ses frères, sous la direction du même Visconti.
} 
brillante sous le ciel - mais dont la dimension fantasmatique est évidente : paysage de rêve, projection d'un état d'âme. Les aspects les plus réalistes du décor: le détail des deux intérieurs avec mobilier et objets, les bruits d'une exactitude documentaire (une bande enregistrée à New-York où alterne le son d'une ville portuaire et celui d'un quartier central selon que la scène se passe chez l'un ou l'autre des deux personnages, défile en continu pendant toute l'action) sont intégrés dans un univers flou et vaporeux (de grands panneaux de tulle peints esquissent les limites incertaines des deux appartements). L'ensemble paraît sortir d'un rêve... » ${ }^{11}$

Visconti avait déjà utilisé des grands voiles de tulle ${ }^{12}$ pour les décors de Notti bianche («Les Nuits blanches », inspiré de Dostoïevski), tourné en 1957 dans les studios de Cinecittà qui reconstituaient une atmosphère de brumes dans un port inspiré de celui de Livourne ${ }^{13}$. Outre la partition de la scène en deux lieux distincts et éloignés, Visconti reprend de Gibson le découpage :

\section{Acte I}

Scène I - Les deux pièces au soir d'un jour de septembre.

Scène II - Le living- room de Gittel, à midi le même jour.

Scène III - Les deux livings-rooms à l'aube du jour suivant.

Acte II

Scène I - Le living-room de Jerry, au crépuscule en octobre.

Scène II - Les deux livings-rooms, à midi, en décembre.

Scène III - Le living-room de Gittel, pendant la nuit d'un samedi de février.

\section{Acte III}

Scène I - Le living-room de Gittel, à midi au mois de mars.

Scène II - Le living-room de Jerry au crépuscule d'un jour de mai.

Scène III - Les deux livings-rooms, quelques jours plus tard, dans l'après-midi.

Trois espaces scéniques fixes sont proposés (pas de plateaux tournants comme chez Gibson-Penn) et les espaces doubles (les deux appartements en présence) définissent le lieu

\footnotetext{
${ }^{11}$. Marie-Madeleine Mervant-Roux, « Scénographie pour une ville qui se perd. France : années cinquantequatre-vingt », in Images de la ville sur la scène au XIXe et XX $X^{e}$ siècles, Paris, Éditions du CNRS, 1991, pp. 183234, p. 198. L'auteur illustre son article par différentes photographies de décors de mise en scènes, dont une de Deux sur la balançoire.

${ }^{12}$. Cf. le croquis d'implantation du décor sur calque qui fait état des jeux de tulle sur plusieurs plans. Cote : 1TMS-00008 (RES).

13. Marcello Mastroianni, Maria Schell et Jean Marais en étaient les interprètes principaux.
} 
des échanges téléphoniques entre les deux protagonistes. D’une parfaite symétrie, le découpage met l'accent sur la scène centrale de l'Acte II. Celle-ci constitue la clé de voûte de l'ensemble, elle en est le point climax : les échanges téléphoniques se démultiplient et débordent les seuls deux personnages. Interviennent des personnages extérieurs dont Tess, qui rompent le lien exclusif, ce fil ténu que Gittel et Mosca ont tenté de tisser et de maintenir. Les sonneries de téléphone polluent, envahissent l'espace sonore. Cette scène est le lieu de retournement, on sait que le couple va se défaire. Situées de part et d'autres, les scènes I et III de l'Acte I, et l'ultime scène sont fondées sur ce principe d'échanges téléphoniques et épousent le parcours narratif très simple de la pièce (début - milieu - fin) : rencontre obstacle - adieu. Ou pour reprendre à dessein une terminologie musicale : anacrouse - accent - désinence. La désinence, très brève, expéditive, se réduit à un court dialogue entre les deux personnages, à une «liquidation des thématiques» ${ }^{14}$. La pièce s'achève sur une dernière sonnerie de téléphone interrompue (Gittel raccroche brutalement, Jerry, sur le pas de la porte, hésite et part).

Enfin notons ce motif ajouté par Visconti : le camélia qui ponctue et symbolise l'histoire entre Gittel et Jerry. Jerry le subtilise, dès la deuxième scène, dans la chambre de Gittel, puis n'a de cesse tout au long de la pièce de le prendre, de le humer, de le laisser pour finalement l'oublier ; celui-ci se fane. Allusion à la Traviata, mise en scène par Visconti qui dirige Maria Callas, si ce n'est que l'héroïne de Gibson est affectée d'une moins noble et plus moderne maladie.

\section{Musiques et intersections}

L'une des particularités de la mise en scène de Visconti est d'avoir imaginé une bande-son qui diffuse en continu des morceaux de musique en alternance avec des bruits de la ville de New-York et autres adjuvants sonores captés par Fred Kiriloff ${ }^{15}$.Voici la liste et le minutage des musiques telles qu'elles figurent dans le dossier de mise en scène avec les références d'enregistrements :

\footnotetext{
${ }^{14}$. Nous reprenons des termes techniques musicaux, à même de décrire et de saisir la fonction structurelle de la pièce dans ses éléments thématiques et leur agencement.

${ }^{15}$. A 20 ans, Fred Kiriloff (1928-2012) «entre en coulisses » selon son expression grâce à Roger Hart. Il fait ses classes d'ingénieur du son à la Radio notamment avec Etienne Bierry. Il travaille avec Raymond Rouleau, Peter Brook, Visconti, Jean Mercure, Jean-Marie Serreau, François Maistre, Jean Le Poulain, etc. Qualifié de magicien du son, il est un pionnier au théâtre et notamment de l'émission «Au théâtre ce soir ». En 2012, peu avant sa mort, il fait don à Danielle Mathieu-Bouillon et à l'ART de toutes ses archives. Ce fonds n'est toujours pas disponible ; en revanche, Olivier Morel a numérisé, pour notre recherche sur le spectacle Deux sur la balançoire, les bandes magnétiques.
} 
Acte I

Body and Soul (Colemann Hawkins) R.C.A 430.234 / LPM 1281 - 3’ 50”

Sonate pour clavier (Scarlatti) N433 « Pastorale » Discophiles français DF 140 - 4’30"

Concerto en sol majeur (Ravel) Pathé Vox. PL 9220 - 5' 30"

Scènes d'enfants (Schumann) Discophiles français DF 128 - 2' 20"'

Acte II

West end blues (Gene Norman) Vogue EPL 7275 - 4'45”

Concerto en sol majeur (Ravel) Pathé Vox. PL 9220 - 9’ 55”

Acte III

Juke Box Special (Ray Anthony) Capitol F 3029 - 0'25"

Alone Together (Barclay 34.042) - 3' 50"

Le choix des musiques, leur minutage et leur enchaînement dessinent, à l'instar de la structure de la pièce, une forme close sur elle-même. Ces musiques nous parviennent par la radio dont Gittel manipule le bouton de variation de volume, tout au long du premier Acte, avant qu'elle ne la prête à Jerry. On ouvre sur du jazz et l'on referme sur du jazz : le jazz serait la part concrète, active, pulsée, comme si la vie à New-York nous était contée. En son centre, la musique de tradition européenne déploie, à rebours, une dimension onirique, un « ineffable », avec la place toute singulière tenue par le Concerto en sol majeur de Ravel. Du minutage on déduit que c'est le $1^{\mathrm{er}}$ mouvement qui est entendu lors du premier Acte, entouré par du Scarlatti et du Schumann. La sonate La Pastorale de Domenico Scarlatti impose un espace musical tonalement et thématiquement stable, répondant au format ternaire général de la pièce, ramené ici à 4 minutes selon la découpe traditionnelle : A(A)BA. La «Rêverie » (déduite du minutage également) tirée des Scènes d'enfants de Schumann pourrait évoquer la part enfantine et naïve du personnage de Gittel et du sentiment paternel, paternaliste que Jerry éprouve à son égard. La pièce se clôt sur la dernière réplique de Jerry : «Je t'aime, petite fille ». Avec sa mélodie lente et étirée, presque incertaine, qui jamais ne semble pouvoir finir, le deuxième mouvement du Concerto en sol majeur ouvre une large brèche de suspens et de rêve. Ces dix minutes, détachées du contexte new-yorkais, occupent toute la première scène du deuxième Acte ; elle sont une sublimation de l'histoire de ce couple, Jerry est alors « aux commandes » de la radio. L'épisode ravélien cède aux bruits de la ville qui perdurent, entêtants, jusqu'à la fin de la pièce avant que ne sonne « Alone together ».

Et le rideau tombe sur les bruits, la rumeur de la ville qui a tout englouti. 
« À partir des années 1930, les matériaux enregistrés se sont substitués lentement (Jean-Marc Larrue parle de «résistance médiatique »), mais sans solution de continuité, aux productions des bruiteurs. L'invention du magnétophone a accéléré cette évolution. Ainsi, c'est pour le spectacle Deux sur la balançoire [...] qu'a été réalisée une des premières véritables bandes-son de l'histoire du théâtre français ${ }^{16}$.

En 1954, Fred Kiriloff propose sa première « véritable » bande-son ${ }^{17}$ pour l'adaptation à la scène par Thierry Maulnier du roman d'André Malraux La Condition humaine, dans une mise en scène de Marcelle Tassencourt au Théâtre Hébertot. La désignation « décor sonore » laisse place à celle de «création sonore ${ }^{18}$ : Deux sur la balançoire après La Condition humaine constitue une étape décisive dans la réévaluation du statut du son au théâtre.

\section{L'écoute pure}

La bande magnétique de Deux sur la balançoire est composée de neuf plages sonores :

1- Ambiance rue portuaire

2- Rue avec Passage de voitures

3- Cloches

4- Carillon et cloches

5- Publicité radio pour le New York Times

6- Cloche de gare

7- Clochette

8- Sirène de pompier

9- Autre sirène de pompier

Chacune des plages peut se qualifier comme un paysage sonore. Un paysage sonore se caractérise par un timbre, un grain, une tonalité générale, «il désigne spécifiquement ce qui dans un milieu sonore est perceptible comme unité esthétique ${ }^{19}$. Autrement dit, pas de

\footnotetext{
${ }^{16}$. Marie-Madeleine Mervant-Roux, « De la bande-son à la sonosphère. Réflexion sur la résistance de la critique théâtrale à l'usage du terme «paysage sonore » », Images Re-vues, $\mathrm{n}^{\circ}$ 7, Paysages sonores, 2009, p. 80.

${ }^{17}$. Cf. Antoine Andrieu-Guitrancourt et Serge Bouillon, Jacques Hébertot le magnifique, Paris bibliothèques, Fondation Hébertot, 2006 : les pages 230-231 consacrées à la création de La Condition humaine et au montage sonore de Fred Kiriloff.

${ }^{18}$. Cf. Marie-Madeleine Mervant-Roux, «De la bande-son à la sonosphère. Réflexion sur la résistance de la critique théâtrale à l'usage du terme «paysage sonore » , op. cit., p. 82. «Élaborer ce qu'on appelle désormais «le son»d'un spectacle devient un vrai métier, une compétence technique spécifique, et une dimension importante de la mise en scène à une époque où mettre en scène ne signifie plus seulement diriger des comédiens mais maîtriser l'ensemble des éléments scéniques à partir d'un point de vue unique. " Notre travail, disait Serré, est un art d'accompagnement ». C'est d'une part parce que Serré, Bergel, Cacchia, Deshays, etc. ont réinventé, de spectacle en spectacle, la relation du visible et de l'audible au théâtre, devenant ainsi eux-mêmes des inventeurs et des artistes, mais aussi parce que le son ne s'est jamais tenu tranquille dans son statut décoratif... ». 19. Jean-François Augoyard et Henri Torgue, À l'écoute de l'environnement. Répertoire des effets sonores, Marseille, Editions Parenthèses, 1995, p. 8. Cf les travaux de Murray Schafer.
} 
conception bruitiste à l'œuvre, mais du son que l'on pense et que l'on compose. Cependant ces paysages sonores captés par Fred Kiriloff de la ville de New York ne sont pas tous de même nature et conséquemment occupent des fonctions précises. En schématisant, il y a ceux qui relèvent de la rumeur sonore à proprement parler, désignés comme «bruits de ville ${ }^{20}$ : les plages 1 et 2 campent les ambiances sonores de chacun des appartements; et ceux qui accompagnent et ponctuent le drame et son déroulement : ils sont à fonction narrative. Il s'agit des plages 3 à 9 qui interviennent à des moments précis et se superposent aux deux rumeurs fondamentales. Celles-ci désignées comme « Ambiance rue portuaire » et « Rue avec Passage de voitures », se superposent ou sont en alternance selon que les scènes se passent chez l'un ou chez l'autre, ou les deux. Soulignons que la fonction narrative cède ou très vite s'estompe pour laisser place à l'écoute pure. Les plages 3 et 4 «Cloches » et «Carillon et cloches » ne renvoient pas seulement à l'appartement de Jerry et à son identification (celui-ci vit près de l'église Saint-Patrick et les cloches sonnent régulièrement et parfois à toute volée $^{21}$ ), elles sont un moment délectable qui n'est pas sans rappeler les belles pages que Gaston Bachelard ${ }^{22}$ a dédiées à l'écoute des cloches de son village (en oubliant le nombre de coups).

On pourrait dresser une courbe générale sonore et l'on verrait se dessiner une forme en arche où le point climax est atteint à la scène centrale du deuxième Acte : là se pressent et s'accumulent les bruits de toutes sortes et de toutes provenance au-delà de ce double socle constitué des deux rumeurs fondamentales. A cela s'ajoute l'élément sonore clé sur lequel est basé le drame : le téléphone et cette démultiplication à l'œuvre dans la scène centrale (comme déjà mentionné). Les sirènes de pompiers sont également un signal très fort ${ }^{23}$, elles annoncent et précipitent les événements, semblent y contribuer - l'effet de tension est alors palpable, et l'on s'achemine vers l'ultime confrontation (querelle) et la rupture

C'est sur ce fond sonore, cette rumeur sonore parfois différenciée et articulée (par superpositions de strates), que s'enlèvent les musiques. Ce fond est le socle, « un canal de

\footnotetext{
${ }^{20}$. La pièce de Gibson mentionne les bruits de ville sous le terme «TRAFFIC ».

${ }^{21}$. Acte I : « les bruits de la ville redémarrent avec le $3^{\mathrm{e}}$ tableau. Les cloches de St Patrick ont sonné les 5 coups chez lui ». p. 51. Cf. Cote : 4-TMS-03383 (RES)

${ }^{22}$. Gaston Bachelard, La poétique de la rêverie [1960], $7^{\mathrm{e}}$ édition, Paris, Presses universitaires de France, «Quadrige », 2011.

23. Acte III, scène II :

p. 24, Gittel ouvre, parmi des lettres de facture, etc., la lettre bleue, qui est parfumée. Pompiers sirène.

L'enveloppe jaune la tente, se décide à l'ouvrir.

p. 25, elle commence à lire à haute voix : c'est l'acte de divorce qui a été prononcé. Jerry qui était à la cuisine dit lentement la suite qu'il connaît par cœur. Pompiers sirène. Cote : 4-TMS-03383 (RES)
} 
communication ${ }^{24}$ comme le désignait naguère Michel Serres. Forme(s) sur fond: les musiques choisies par Visconti offrent des points d'articulation, des signaux ${ }^{25}$ à la bande qui diffuse en continu. Le relevé de mise en scène en précise les entrées et les bruits de ville peuvent alors s'éloigner ou s'éteindre. Autrement dit, il y a alternance, plus précisément tuilage, « fondu-enchaîné » ${ }^{26}$.

Nos deux comédiens vont donc évoluer, parler et dialoguer, moduler leur voix, en modifier le débit, les courbes intonatives, sur ce socle sonore-musical qui jamais ne se tait. Les conditions de diction en sont modifiées et dans le droit-fil les conditions d'écoute du spectateur en seront peut-être bouleversées. On inaugure une troisième écoute ${ }^{27}$ sur un canal tri-dimensionnel (la bande, les musiques, les voix), qui gomme les frontières et les hiérarchies : que doit-on écouter, que doit entendre ? Que signifie écouter?

\footnotetext{
${ }^{24}$. Michel Serres, Esthétiques sur Carpaccio, Paris, Le Livre de Poche, 1995 [Hermann, 1975], p. 145.

${ }^{25}$. Notion introduite par Pierre Boulez assortie de sa notion complémentaire d'enveloppe. «Un signal est, en quelque sorte, une réduction de la thématique à un élément plus fort qui absorbe tous les autres : signal fixe, signal variable, il indique d'une façon tellement forte qu'il peut acquérir une signification par lui-même... ». « Si les signaux sont des phénomènes ponctuels, marquant l'articulation ou la discontinuité, les enveloppes, quant à elles, ont un caractère global. Elles marquent la prévalence, pour un temps, d'une dimension thématique par rapport aux autres... », Jalons pour une décennie, Paris, Christian Bourgois éditeur, 1989, p. 269.

${ }^{26}$. Quelques exemples, écrits à la main, tirés du premier acte : « Fondu-enchaîné - lumière Radio - le $2^{\mathrm{e}}$ Tableau commence sur des bruits de ville » (p. 19); du deuxième acte : «Elle allume le poste et progressivement les bruits de ville disparaissent » (p. 32) : 4-TMS-03383 (RES)

${ }^{27}$. Cf. Roland Barthes, «Écoute », op. cit.
} 